\title{
Controversial report finds no proof that dental braces work
}

A recently published report ${ }^{1}$ commissioned by the German government has concluded that there is no evidence that orthodontic treatment works.

The controversial finding has been played down by the government which said it was 'unsatisfactory' and the British Orthodontic Society (BOS) has stressed that there are many studies that suggest quality of life is significantly poorer in people with malocclusions, compared with those without.

Germany's Federal Ministry of Health commissioned the IGES Institute to carry out a review (meta-analysis) of orthodontic treatments which reported back in November 2018 and the Ministry responded on 4 January 2019.

The review's authors were asked to examine:

- What long-term effects do the most important orthodontic treatments have on oral health

- What are the financial costs of the statutory health insurance and the self-payer for orthodontic services

- What further research needs to exist to determine the benefits of orthodontic treatment.

The report authors concluded that the data basis was currently insufficient to conclusively assess the benefit of orthodontic treatment and that there was no evidence to back claims that braces provided long-term dental health benefits.

The authors said: 'As a whole, the studies into diagnostic and therapeutic orthodontic measures allow no conclusion to be drawn about a patient-relevant benefit.' They also said it was 'noticeable' that no long-term studies had collected data on the impact of orthodontic treatments on tooth loss or secondary diseases.

'All in all, the identified studies regarding the diagnostic and therapeutic orthodontic measures do not allow any conclusions about a patient-relevant benefit.

'This is due in particular to the heterogeneity of the studies in terms of the populations considered, the interventions used and the study designs, and to the fact that morbidity-relevant outcomes such as tooth loss, tooth decay or periodontitis and periodontitis usually occur only several years after the treatment and thus very much require long observation times.'

However, they also found that 'patients with a completed orthodontic treatment reported a higher quality of life than untreated study participants who are currently undergoing orthodontic treatment'.

A Ministry spokesperson said: 'Although it is not possible to prove that the braces reduce morbidity (tooth decay, periodontitis, tooth loss, etc), according to IGES, this is not ruled out.

'However, the authors of the study note that dental malocclusion and the quality of life of the patients improve as a result of this treatment. In principle, the benefit of a therapy is not assessed by the legislature, but by the Federal Joint Committee. The Ministry will discuss further research needs and further recommendations for action with the organisations involved.'

Peter McCallum, Director of External Relations for the BOS, told the $B D J$ : 'It is important that we consider the social, emotional and functional handicaps that produce a significant need for orthodontic Treatment in addition to purely dental health benefits.

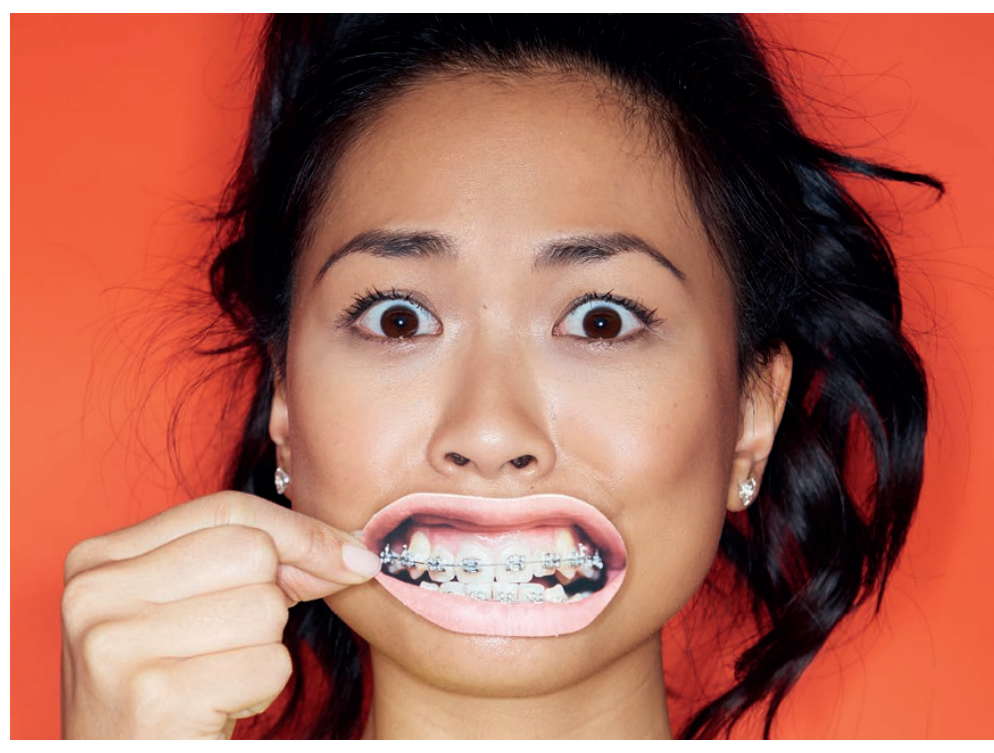

'The WHO [World Health Organisation] definition of health is as "a state of complete physical, mental and social wellbeing and not merely the absence of disease or infirmity." Orthodontic treatment can allow individuals to cope more effectively in social situations, without concern for the appearance of their teeth.

'In regard to the IGES Institute study, it is worth noting that the German Health Ministry has deemed the study 'unsatisfactory' with Health Minister Jens Spahn saying he had 'no doubts' about the necessity of orthodontic treatment.'

McCallum referred to the Index of Orthodontic Treatment Need created to assist orthodontists to determine the efficacy and appropriateness of care which consists of a Dental Health Component (DHC) and an Aesthetic Component (AC).

There were various reasons for orthodontics to be provided on DHC grounds, he said, which included:

- Ectopically positioned and unerupted teeth

- Missing teeth

- Increased overjet (may be subject to damage through trauma)

- Reverse crossbites with functional difficulties

- Cranio-facial abnormalities, including cleft lip and palate

- Supernumerary teeth.

'Clinical experience suggests that having poorly aligned teeth may reduce the potential for natural tooth cleansing and increase the risk of tooth decay. Malocclusion could thereby contribute to both dental decay and periodontal disease, which would damage the long-term health of the teeth and gums as it makes it harder for the patient to take care of their teeth properly,' said McCallum.

'A number of systematic reviews are now available, looking at the quality of life impacts of having a malocclusion and, although there remains heterogeneity in the studies included, the reviews have all concluded that quality of life is significantly poorer in those with malocclusions, compared with those without.'

1. IGES Institute. Orthodontic Treatment Measures. 2018. Available at https://www.bundes gesundheitsministerium.de/fileadmin/Dateien/5_Publikationen/Praevention/Berichte/ IGES_Gutachten_KfO.pdf (accessed January 2019). 